\title{
How Cancer Shapes Evolution and How Evolution Shapes Cancer
}

\author{
Matias Casás-Selves • James DeGregori
}

Published online: 2 December 2011

(C) Springer Science+Business Media, LLC 2011

\begin{abstract}
Evolutionary theories are critical for understanding cancer development at the level of species as well as at the level of cells and tissues, and for developing effective therapies. Animals have evolved potent tumor-suppressive mechanisms to prevent cancer development. These mechanisms were initially necessary for the evolution of multi-cellular organisms and became even more important as animals evolved large bodies and long lives. Indeed, the development and architecture of our tissues were evolutionarily constrained by the need to limit cancer. Cancer development within an individual is also an evolutionary process, which in many respects mirrors species evolution. Species evolve by mutation and selection acting on individuals in a population; tumors evolve by mutation and selection acting on cells in a tissue. The processes of mutation and selection are integral to the evolution of cancer at every step of multistage carcinogenesis, from tumor genesis to metastasis. Factors associated with cancer development, such as aging and carcinogens, have been shown to promote cancer evolution by impacting both mutation and selection processes. While there are therapies that can decimate a cancer cell population, unfortunately cancers can also evolve resistance to these therapies, leading to the resurgence of treatmentrefractory disease. Understanding cancer from an evolutionary perspective can allow us to appreciate better why cancers predominantly occur in the elderly and why other conditions, from radiation exposure to smoking, are associated with increased cancers. Importantly, the application of evolutionary
\end{abstract}

M. Casás-Selves $\cdot$ J. DeGregori $(\triangle)$

Department of Biochemistry and Molecular Genetics,

Department of Immunology, Department of Pediatrics,

Program in Molecular Biology,

University of Colorado School of Medicine,

Aurora, CO 80045, USA

e-mail: james.degregori@ucdenver.edu theory to cancer should engender new treatment strategies that could better control this dreaded disease.

Keywords Tumorigenesis · Cancer evolution · Tumor suppression · Adaptation

\section{Why Understanding Cancer from an Evolutionary Perspective is Important}

We expect that the public generally views evolutionary biology as a science about the past, with stodgy old professors examining dusty fossils in poorly lit museum basements. Evolution must certainly be a field well separated from modern medicine and biomedical research, right? If the public makes a connection between evolution and medicine, it is typically in the example of bacteria acquiring antibiotic resistance. But what does evolution have to do with afflictions like heart disease, obesity, and cancer? As it turns out, these diseases are intricately tied to our evolutionary histories, and understanding evolution is essential for preventing, managing, and treating these diseases (Greaves 2007; Nesse 2001). This review will focus on cancer: how evolutionary theories can be used to understand cancer development at the level of species as well as at the level of cells and tissues. We will also discuss the implications and benefits of an evolutionary perspective toward cancer prevention and therapies.

\section{Life History, Lifespan, and Cancer}

For almost all animals, old age is associated with a general decline in tissue structure and function. This decline is thought to reflect the lack of selective pressure to maintain tissues beyond an age when the animal would be likely to contribute genetically to future generations (Kirkwood 2005; Medawar 
1952; Williams 1957). Similarly, there is little selective pressure to limit cancer in old animals who are substantially beyond their reproductive years (DeGregori 2011). For example, while mice can live two to four years in the lab and tend to develop cancer in their second and third years, it is rare to find a mouse greater than one year old in the wild (Kirkwood 2005). Most wild mice will be dead from other causes such as cold, hunger, disease, or predators well before the age when cancer would be a likely cause of their demise. Thus, evolution has favored a "breed early, breed often" strategy for mice. Investment in better tissue maintenance or cancer prevention well after one year would have required allocation of precious energy early in life, when this energy would be better spent on survival and reproduction during youth.

The situation in humans is more complex, as even our hunter-gatherer ancestors may have had a reasonable chance of living past 50 once they survived to adulthood (Kaplan et al. 2000). It is notable however that survival into older adulthood was probably much lower prior to the
Upper Paleolithic (10,000-40,000 years ago; Caspari and Lee 2004). Regardless, the chances of older humans contributing to the gene pool of future generations should have declined with age, as an earlier demise due to disease, starvation, predators, or other causes became more likely (and for females, the chances of successfully raising offspring became less likely). Thus, evolutionary investments in tumor suppression may have waned in older age.

So while George Bernard Shaw remarked that "Youth is wasted on the young," "youthfulness" (and fitness; see Box 1 for definitions of italicized words) is maintained precisely where it is needed: during the period when reproductive success is most likely. Moreover, some processes which are important for organismal fitness in youth may actually contribute to tissue decline and increased cancer in old age, a concept known as antagonistic pleiotropy (Campisi 2005). Thus, evolution has in effect weighed the costs and benefits of somatic tissue maintenance and tumor suppression, favoring a strategy that maximizes reproductive success.

\section{Box 1: Glossary:}

Adaptive- increases fitness (e.g. a mutation that increases cellular fitness would be adaptive) Antagonistic pleiotropy- a gene or trait which is advantageous during youth but contributes to aging phenotypes.

Carcinogen- any agent (chemical, physical or biological) that can directly or indirectly cause cancer

Cell fitness- a measure of the ability of a cell to pass its genotype on to future cell generations; cell fitness is not simply a measure of cell duplication rates, as a stem cell will often need to limit cell duplication rates to maintain itself within the stem cell niche (necessary to remain a stem cell)

Cell senescence- a permanent elimination of the proliferation potential for a cell

Growth and Survival Factors- small proteins that can pass between cells to confer increased cell proliferation and survival on the recipient cells.

Immortal cells- a clone of cells with unlimited ability to divide and generate cell progeny.

Malignant- abnormal tumor growth within a tissue that exceeds normal tissue boundaries, potentially causing damage to the tissue or organ

Metastasis- the process whereby cancer cells leave their tissue of origin and establish new tumors in additional sites in the same or other organs

Niche- for a stem cell, this refers to the environmental factors (other cells, matrix and soluble factors) that influence the properties of the cell

Oncogene- a gene whose dysregulation or mutational activation can contribute to cancer development (i.e. cancer promoting genes) 
Oncogenic mutation- a change in the DNA code of a normal cellular gene that creates an oncogene or tumor suppressor gene

Organismal fitness- a measure of reproductive success (the ability of an organism to pass its genes on to future generations of that organism)

Somatic- cells and tissues of an organism, excluding gametes (e.g. sperm and eggs)

Stem cells- the small population of cells that maintain a tissue throughout the lifespan of an animal. These cells are at the tip of a cellular hierarchy, with cells that are more committed to clearly defined roles specific for the tissue further down the hierarchy.

Telomeres- structures at the ends of chromosomes which protect these ends. Telomeres shorten with each cell division unless maintained by an enzyme called telomerase.

Tumor Suppressor gene- a gene whose inactivation (such as by mutation) can contribute to cancer development (i.e. cancer suppressive genes)

\section{Evolved Tumor Suppression}

Just as organisms evolve, tissue (somatic) cells can also evolve within an animal. Somatic cell evolution, which can lead to tissue-disrupting tumors and cancer, is clearly detrimental to the fitness of the organism (with the exception of cell evolution within the immune system). The reason that cancer is so rare during youth is that multi-cellular organisms, particularly longlived and large ones, have evolved potent tumor-suppressive mechanisms (but see Box 2 for evolutionary explanations for childhood cancers). Indeed, the evolution of multi-cellularity required it. We can imagine that the first multi-cellular organisms, no matter how simple, would not have been able to evolve significant organization or complexity without mechanisms to avoid rogue cell growth. But how could a group of cells representing a recently evolved multi-cellular organism have prevented one of their own from acquiring a short-term advantage (i.e. rogue cells, out-proliferating their peers), even if it was to the eventual detriment of the organism? Successful multi-cellular organisms evolved mechanisms to limit these nonconformist outgrowths. We will refer to these tumor-suppressive mechanisms as either intrinsic (how cells avoid becoming tumor cells) or integral (cancer avoidance at the level of tissues and the whole organism).

Box 2: So what about cancer in children? Most childhood cancers result from mutations in oncogenes and tumor suppressor genes acquired either during fetal development or after birth. While cancer is rare in children ( $1 / 1000$ overall), such cancers would be expected to be highly selected against during our evolution, given that until the last half century, these cancers almost inevitably led to death prior to reproduction (thus, these cancers had a very high fitness cost). Several evolutionary rationales have been proposed. First, as noted by Crespi and Summers (2005), recent evolution has substantially altered the human brain, bones and immune system, and a risk of childhood cancers affecting these tissues may have been a tradeoff against the advantages of a more developed brain, faster bone development in adolescence, and a better immune system. Second, Greaves has argued that maladaptation of our ancestral genomes with modern environments, lifestyles, and diets has contributed to recent increases in cancers such as childhood acute lymphoblastic leukemias: Our immune systems did not evolve to deal with modern (hygienic) conditions but to conditions with more pathogen exposures early in life (Greaves 2007). Thus, our hematopoietic systems are not truly adapted to modern life. Finally, the rapid growth of tissues during fetal development and early childhood may simply facilitate the acquisition of cancer-causing (oncogenic) mutations, and the low rate of childhood cancers has not sufficiently impacted human fitness to select for further tumor suppressive innovations (or the cost of these innovations may be greater than their benefit for our species). 
Intrinsic Tumor Suppression

Cells of multi-cellular organisms have evolved mechanisms to maintain appropriate numbers of cells within tissues. Both cell division and cell survival are regulated by various "social" cues including circulating factors and contact with other cells (Hanahan and Weinberg 2011). Importantly, there are often mechanisms to penalize disobedient cells. For example, dividing outside of the appropriate structure, such as a wellordered duct, can lead to cell suicide (a program that is activated within the cell that leads to cell death) (Hipfner and Cohen 2004). These mechanisms are essential for the proper development and function of complex tissues and organs. Animals have also evolved cell suicide and cell senescence responses to inappropriate signals, such as those engendered by cancer-causing (oncogenic) mutations (Lowe et al. 2004). Similar cell death and senescence responses are initiated following cellular damage, particularly damage to DNA. These intrinsic mechanisms contribute to tumor suppression (Lowe et al. 2004) by limiting the chances that damaged, and possibly oncogenic, cells are propagated. In addition, oncogenic mutations are avoided in cells by effective DNA repair (Hoeijmakers 2009), which even unicellular organisms use to maintain the integrity of their genetic material.

Another intrinsic tumor-suppressive mechanism is mediated by telomeres, which cap chromosome ends to help maintain the integrity of the DNA code (Sharpless and DePinho 2004; Chan and Blackburn 2004). Singlecell organisms as well as germ cells of animals maintain telomeres continuously as a means to ensure correct propagation of their genetic code. But somatic cells in adult tissues do not maintain telomeres continuously, and these structures are reduced in length with age, until a critically short length stimulates cell death or senescence (which may contribute to tissue decline with aging). Larger animals appear to particularly favor this mechanism to limit telomere maintenance during aging, which by restricting each somatic cell's lifespan is thought to limit cancer (which requires immortal cells; Gorbunova and Seluanov 2009). Together, all of these mechanisms function within cells to limit their ability to disobey tissue rules, thus reducing the risk of cancer.

\section{Integral Tumor Suppression}

Compared to other animals (and with notable exceptions), vertebrates have longer lives and bigger bodies, providing a bigger pool of cells over longer time periods in which an oncogenic mutation may arise. Consequently, vertebrates should have increased requirements for suppression of rogue cell growth. While enhancements in the intrinsic tumor- suppressive mechanisms discussed above may have contributed to improved tumor suppression in the large and longlived, additional mechanisms operating at the tissue and animal levels clearly function in vertebrates (and to varying extent in other animals) to limit cancer development. These integral tumor-suppressive mechanisms include immunity, tissue organization, and the fitness of stem cell pools.

Vertebrates have evolved effective immune systems, and even invertebrates possess simple immune systems (Robert 2010). In particular, vertebrates have evolved antigenspecific immunity, with lymphocytes that are tailored to recognize and respond to specific foreign or abnormal proteins ("antigens," whether these antigens are part of viruses, bacteria, or cancer cells). In addition to eliminating pathogens and limiting infections, these systems can target precancerous and cancerous cells for destruction, thus contributing to tumor suppression (Robert 2010). Indeed, patients with defective cellular immunity have increased cancer risk (Dunn et al. 2004).

The evolution of animals and their tissues, organs, and systems has been constrained by the requirement to avoid tumors (Cairns 1975; reviewed in DeGregori 2011). These constraints on how tissues develop and are organized should be particularly severe for animals with larger bodies and longer lives, which require tissue maintenance and renewal throughout life. Tissue organization contributes to the "peer pressure" exerted on malignant cells, whereby normal tissue structure can suppress rogue cell expansion (Bissell and Hines 2011). Also contributing to tumor suppression, tissues in vertebrates are often maintained by a hierarchy of cells, with a small number of stem cells serving as the source of the much more numerous specialized cells that actually carry out the functions of the tissue (Weissman 2000). Stem cells are thought to often represent the targets for cancer initiation (Reya et al. 2001). These cells are maintained throughout life, increasing the opportunities for mutation accumulation. Importantly, hierarchical tissue organization, with a few stem cells at the top of the hierarchy, would reduce the pool of cells most susceptible to oncogenic mutations. Stem cells in tissues also appear to be positioned in protective locations (their niche). For example, blood stem cells are localized in the bone marrow, and gut stem cells are positioned distant from the nasty contents of the large intestine (Cairns 1975; Reya et al. 2001; Gatenby et al. 2010), which should reduce exposure to carcinogens. Most cell proliferation is delegated to non-stem cells (which are derived from stem cells) in the hierarchy, and these cells are generally short-lived. Indeed, for the intestines, these non-stem cells (even if they acquire an oncogenic mutation) are destined for a one-way trip out the anus (hence mutations get "flushed") (Frank and Nowak 2004; Pepper et al. 2007). Thus, vertebrates have evolved effective strategies to minimize cancer rates, despite a 
dizzying number of cell divisions required daily to maintain some organs like the blood, skin, and intestines throughout life. For example, each human produces around $10^{11}$ (one trillion) blood cells each day (Gordon et al. 2002). There were probably other possible approaches to the development and maintenance of vertebrate organs, but each successful solution needed to be compatible with sufficiently low rates of cancer.

The fact that cancer has exerted developmental constraints during evolution provides another reason that cancer biologists need to understand evolutionary biology, and all biologists need to learn about cancer: Just as one cannot really understand cancer without understanding how evolution has limited it, one cannot fully understand the evolution of form and function in multi-cellular organisms without considering how the requirement for tumor suppression has constrained it. So cancer biology courses should not just be for medical students, and evolutionary biology should be required for all life science disciplines, including medicine. Teaching students about cancer from an evolutionary perspective would not only enhance the students' understanding of cancer, but would provide highly relevant examples for why evolutionary biology is so important. Students should learn that fighting cancer requires an understanding of its evolutionary origins and how it adapts to current therapies to the detriment of cancer patients.

\section{Integral Tumor Suppression by Maintaining Tissue Fitness}

Cancer progression occurs by a process of somatic cell evolution whereby a cell clone acquires a number of genetic changes over time and proliferates to generate a highly complex cancer (DeGregori 2011; Merlo et al. 2006; Bagby and Fleischman 2011). This evolutionary process is driven by two major forces:

1) genetic variation in somatic cell populations, which facilitates the acquisition of mutations in oncogenes and tumor suppressor genes;

2) selection for cells that harbor mutations increasing their cellular fitness relative to competing cells.

Certain cells in a tissue with potential to proliferate, like stem cells, are in competition with each other for nutrients, growth factors, survival factors, and spatial locations (Baker 2011; Fleenor et al. 2010), which together constitute their niche. How fit the competing cells are, as well as the state of the niche, can in a large part determine whether an acquired oncogenic mutation leads to expansion of the cell clone. Thus, consideration of cancer development as an evolutionary process within an individual provides a different perspective relative to the more widely accepted focus on oncogenic mutations as the primary drivers of cancer.
We have proposed that long-lived multi-cellular organisms have evolved highly fit stem cell populations, not only as a means of efficiently maintaining tissues but also because a highly fit cell population should oppose somatic cell evolution (DeGregori 2011). Like animal populations well adapted to their environments, cell populations with high fitness should be resistant to change: The chance that a mutation improves fitness will be much less when there is little room for improvement. Highly effective competition in a young, healthy stem cell population should serve to maintain the status quo, preventing somatic cell evolution. In other words, non-conformist cells are suppressed via competition by their fit neighbors (Fig. 1, top), just as a healthy lawn can limit weed growth.

But when general cellular fitness is reduced, such as with aging or following damaging carcinogen exposure, certain oncogenic mutations can be adaptive (restoring fitness; Fig. 1, bottom). An oncogenic mutation could be adaptive by circumventing or fixing problems caused by the damage and may be adaptive in a cell under one set of conditions but not another. For example, if a carcinogen causes widespread cell death in a tissue, there will be increased selection for oncogenic mutations that promote cell survival. Damage to the niche, which also occurs with aging or carcinogen exposure, can also increase selection for adaptive mutations. In some ways, these concepts are analogous to the acquisition of antibiotic resistance in gut flora: Antibiotic treatment will select for mutant bacteria resistant to the antibiotic and will also destroy competing bacteria and substantially alter the niche.

So just as evolution of species is driven by mutation and selection, cancer evolution is also driven by both oncogenic mutations and alterations in selective pressures, both of which can result from aging and carcinogenic exposures. For example, smoking not only exposes your lungs to DNA-damaging chemicals that can cause oncogenic mutations (Hecht 2002) but also should dramatically alter selective pressures within the lung: Normal lung stem cells are highly damaged (reducing their fitness), and the environment that these cells are in (their niche) is highly perturbed (remember the black lungs in a jar that you were shown to keep you from smoking?). Damage to the landscape of the lung also leads to compensatory proliferation to fill empty niches (Takahashi et al. 2010), providing increased opportunities for cancers to develop. Massive destruction of the landscape, such as after the gigantic meteor hit off the Gulf of Mexico at the Cretaceous-Tertiary (K-T) Boundary $\sim 65$ million years ago, can lead to new speciation as organisms adapt to the new environment (Raup 1986). The same probably applies within an individual, although somatic cell evolution within an individual is inherently dangerous, as it can lead to cancer. 
Fig. 1 Oncogenic mutations will rarely be advantageous within a population of healthy well-adapted stem cells residing in a healthy niche, leading to loss of the oncogenic clone over time (top). But following aging or damage-induced reductions in stem pool fitness, together with damage or degradation of the niche, oncogenic events that improve fitness should provide selective advantages, leading to expansion of the oncogenic clone over time (bottom). Cellular competition is denoted with arrows, with darker arrows indicating greater competition

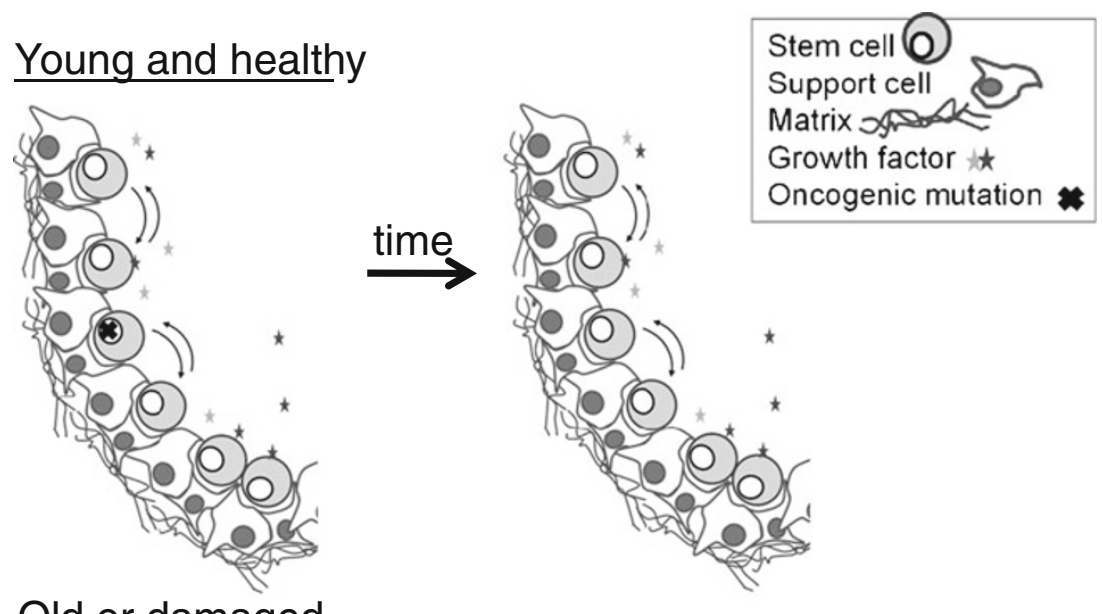

Old or damaged
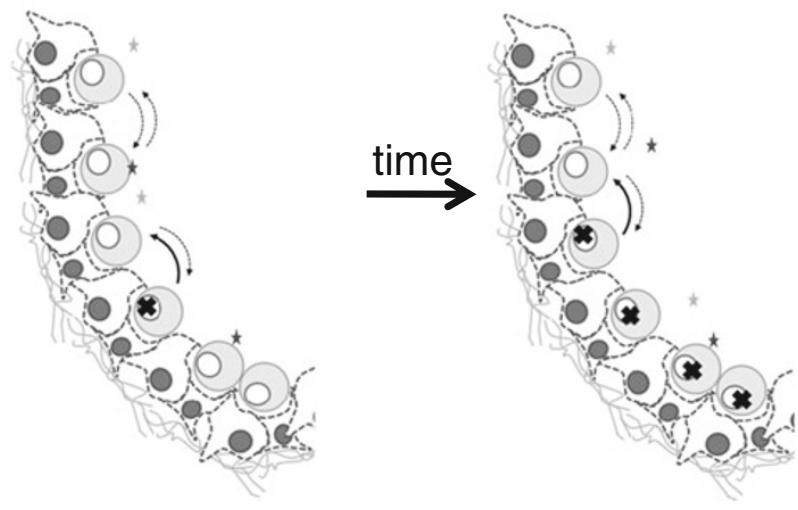

So what lessons can we take from these evolutionary considerations of cancer? For starters, we cannot simply focus on the cancer but need to consider its environment within the body. We need to learn how to attack the cancer and at the same time support the immune system, boost normal cell fitness, and restore a more normal niche. For cancer prevention, maintaining fit tissues by doing all of the things we already know are good for us (eat well, don't smoke, exercise, etc.), should help prevent cancer by maintaining fit cells within a tissue, making it harder for a rogue early cancer cell to outcompete normal cells and expand. The following sections will continue our discussion of cancer as an evolutionary process: How do cells that acquire oncogenic mutations eventually evolve into complex and invasive cancers? How can an evolutionary viewpoint help explain carcinogen-induced cancers? How can an evolutionary understanding of cancer improve therapeutic approaches to combat cancer?

\section{Evolutionary Perspectives on Multistage Carcinogenesis and Metastasis}

The currently accepted oncogenesis model, initially described by Nowell (1976), states that tumors originate from benign growths with minor genetic alterations. Over time, these growths accumulate mutations and advance to more aggressive cancers. In some cases, cancerous cells can disseminate from the original tumor and invade various organs and tissues, a process known as metastasis. Hanahan and Weinberg (2011) have outlined the key biological properties, or hallmarks, of cancer cells, which include: (a) having self-sufficiency in growth signals, (b) loss of sensitivity to anti-growth signals, (c) loss of sensitivity to programmed cell suicide, (d) immortality (cells can divide forever), (e) altered metabolic properties, (f) higher mutation rates, (g) ability to attract blood vessels, and (h) the capacity to invade tissues and become metastatic. So how are normal cells, which are part of a strict tissue organization with cellular social cues, transformed into cellular sociopaths who disregard tissue order and ignore cellular dialog?

Hallmarks of cancer are acquired via mutational changes that provide a potential tumor cell with a fitness advantage relative to normal cells within the same niche, thereby promoting the expansion of the tumor cell clone. As discussed above, whether these mutations confer a cellular fitness advantage should be dependent on the state of the neighborhood (competing cells and the state of the niche). Moreover, just as the presence of any species alters its environment, a 
growing tumor alters its local environment, and thus the parameters that dictate selection for particular mutations change as the cancer develops (Gatenby and Gillies 2008).

Alterations in the local tissue environment progressively create barriers to continued cancer evolution (Gatenby and Gillies 2008), and these barriers are typically effective, preventing further tumor development. Such barriers include oxygen and nutrient limitations that require the acquisition of mutations that promote metabolic changes and/or increase the blood supply. Thus, the hallmarks of cancer described above are the result of cancer evolution as it overcomes selection barriers. These barriers could account for the frequent observation of benign and typically innocuous tumors in tissues in the elderly (Greaves 2007): Most cell clones that start down the path of cancer (fortunately) never progress to the point of clinical relevance (necessitating removal or treatment).

Metastasis is itself a multistage process whereby cancer cells from the original tissue location (the primary tumor) are able to colonize other sites including distant organs (Hanahan and Weinberg 2011). Malignant cells commence this process by local invasion of the organ occupied by the primary tumor. Next, cancer cells escape into either the lymphatic or blood vessel circulation, where they must be able to survive until they can reach a tissue and establish a metastatic tumor growth. Continued growth of metastases in distant organs eventually leads to failure of the colonized $\operatorname{organ}(\mathrm{s})$, and is the primary cause of death for cancer patients. Fortunately, each of these steps, from escape out of the primary tumor, survival in the blood/lymph stream, colonization of a distant tissue, to successful growth of the metastatic tumor in a foreign site, represents a substantial hurdle for the tumor cells. The vast majority of tumor cells will not overcome these hurdles. Only the rare cancer cells possess the appropriate combination of genetic changes and luck (from the cancer's perspective) to initiate a metastatic tumor. Thus, each of these hurdles confers a powerful selective pressure, with selection for particular tumor cell genotypes.

We can envision metastasis in evolutionary terms as an aggressive invasive species (metastatic cancer cells), which migrates into a new environment (organ or tissue), disregarding niche organization and hierarchy. Over time, the invading species takes over this environment and either drives away (out-competes) or kills the native species (leading to organ failure). But why would migratory ability of cancer cells be advantageous in the first place, given that non-migratory cells in the primary tumor might have the advantage that resources could be spent on cell division rather than migration (Bernards and Weinberg 2002)? Indeed, in a homogeneous tumor, migration reduces cancer cell fitness (Chen et al. 2011).
But malignant tumors, particularly ones with metastatic potential, are not homogeneous and instead present diverse environments for cancer cells. Using ecological principles from migratory animal populations, modeling studies have shown how migratory ability, by increasing the ability of a cancer clone to sample different environments with variable resources in the primary tumor, can provide a selective advantage within the primary tumor by allowing the cancer cell clone to experience more favorable environments (Chen et al. 2011). Thus, understanding cancer from an evolutionary and ecological perspective can shed light on its most deadly manifestation: metastasis.

\section{Carcinogens as Promoters of Somatic Cell Evolution}

Carcinogen is a scary word. Carcinogens are agents that contribute to cancer development, and often attributing carcinogenic potential to a substance can be quite controversial, since whether an agent can cause cancer can be dependent on the test or criteria used (Loeb and Harris 2008). For some carcinogens like cigarette smoking, the case is closed, given incontrovertible epidemiological and laboratory evidence. As discussed above, smoking contributes to lung cancer evolution by both increasing mutation frequency as well as by altering the tissue environment to increase selection for adaptive oncogenic mutations. However, given the traditional focus of cancer biology on mutations, and not selection, the most frequently used tests for carcinogens (such as the Ames test; Mortelmans and Zeiger 2000) only determine whether a substance increases mutations, ignoring potential impacts on selection. The only reasonable method to determine the latter requires animal models, which are much more expensive and require humane considerations, and limiting the numbers of animals used entails application of unnatural doses of the suspected carcinogen. But if we consider that cancer is impeded by a healthy normal tissue environment, then the ability of a suspected carcinogen to perturb tissue structure and health could be used as a surrogate for a "cancer-favoring context" in order to justify more expensive cancer studies.

Carcinogens engender two of the major forces for evolutionary change: increased genetic diversity in a population and alterations of selective pressures. Both natural and synthetic compounds can initiate cancer (Loeb and Harris 2008). Among natural carcinogens, a prime example is the potent microbial toxin Aflatoxin B1, produced by the fungus Aspergillus flavus. Chronic exposure to this toxin, mostly found in stored grains and nuts, has been shown to highly predispose to liver cancer (Farazi and DePinho 2006). Even bacteria are now accepted as cancer-causing agents, with Helicobacter pylori (causative 
of gastric ulcers) increasing stomach cancer risk (Piazuelo et al. 2010). The resulting inflammation may contribute to stomach cancer risk both by increasing DNA-damaging agents in the tissue as well as by creating an abnormal tissue environment favoring malignant cancer evolution. We are also exposed to intrinsic carcinogens, such as oxidative products of metabolism, which can contribute to DNA mutations (together with the intrinsic error rate of our cell's DNA replication and repair machinery) (Liou and Storz 2010). Thus, even if we avoided any contact with man-made chemicals, we would still be exposed to carcinogens.

Over the last hundred and fifty years, there have been colossal strides in different areas of science and society which have been translated into massive technological advances. As a by-product of these changes, Homo sapiens currently encounter a plethora of new chemical and physical agents that were not present in nature throughout our long evolutionary history. For example, early humans (and our non-human ancestors) did not encounter any of the synthetic organic molecules we are currently exposed to such as vinyl chloride, dioxins, or benzene (all shown to cause cancers; Loeb and Harris 2008). Consequently, humans did not evolve effective means of protecting their bodies from these novel insults. Even though our enzymatic detoxification systems can still recognize functional groups within these molecules, they are not completely efficient in guarding our bodies from the onslaught of foreign molecular insults. This is especially evident in industrial workers who are exposed to new compounds, resulting in higher incidences of diseases like cancers. Modern humans are also exposed to amounts of ionizing radiation (like $\mathrm{X}$ and gamma rays), whether resulting from nuclear fission or medical use, which far exceed natural levels experienced by our ancestors (Fleenor et al. 2010; Little 2000). Another prime example is modern cancer chemotherapy, where patients are inundated with cytotoxic therapies that not only kill cancer cells but impinge on the health of normal tissues (even leading to new cancers that emerge years later; Allan and Travis 2005). Thus, we must consider that we did not evolve to deal with these carcinogenic insults, which not only increase mutation rates but also can alter selective pressures (thus promoting somatic cell evolution).

\section{Viewing Cancer Therapies Through an Evolutionary Lens}

At this point, hopefully you can now envision cancer as a disease heavily dependent of evolutionary principles and ecological relationships (Merlo et al. 2006). An advanced cancer is a microcosm of cells with ample genetic variability where each cancer cell clone can be considered a competing (or sometimes cooperating) sub-species. This genetic variability can be attributed to the often higher mutation rates, multiple selective hurdles, variable microenvironments, and increased numbers of cell generations that characterize cancers. Cancers consist of from a billion to a thousand billion $\left(10^{12}\right)$ cells, resulting from hundreds to thousands of cell generations (Siegmund et al. 2009), which is on the scale of the number of generations spanning the entire evolutionary history of $H$. sapiens. And just as for animal populations, this great genetic variability in the cancer population provides ample opportunities for adaptation to change and the evolution of diverse types (Marusyk and Polyak 2010). Selective pressures, whether exerted by lack of nutrients, radiation exposure, or chemotherapy, can select for clones that are most adaptive to these conditions and can create an evolutionary bottleneck whereby the selected cells populate the majority of the tumor. We can also consider the hosts' immune system as a predatory selection force which may counteract tumor growth but also select for clones that can "tame" the immune system (and even subvert immune cells for its own nefarious means; Dunn et al. 2004).

Since the origins of oncology, systemic cytotoxic chemotherapy has been the primary strategy for patients with advanced cancers (Mukherjee 2010). While we find a plethora of chemotherapy drugs in use in the clinic, their common fundamental role is to either kill cancer cells or stop their growth potential. With some exceptions like childhood leukemia, this approach has rarely led to cures for disseminated cancers. Although it is understood that cancer is a complex and dynamic disease, it is currently treated according to fixed, linear protocols. Patients are treated with the highest tolerable chemotherapy dose, with the goal of killing the maximum amount of cancer cells. But tumors are highly adaptive and dynamic systems that can evolve strategies to overcome chemotherapy. Although cytotoxic treatment can initially cause a major reduction of the tumor size, this also creates powerful pressure that will frequently select for clones that have intrinsic resistance to the regimen. This evolutionary ability of the tumor cells thus results in regimen failure and regrowth of the cancer, populated by resistant cells (Fig. 2, top).

Mathematical modeling, with model testing in mice, suggests that chemotherapy-sensitive cells in the tumor are often more fit and actually suppress the growth of the less fit chemotherapy resistant clones (Gatenby et al. 2009). Thus, there is probably a fitness cost to chemo-resistance. These researchers envision a mode of cancer therapy which adapts to the predominating species in the tumor: Treat and debulk the tumor with chemotherapy but still maintain a chemo-sensitive population to oppose the growth of chemoresistant clones. Thus, the treatment would cause the tumor size to follow a sinusoid growth curve (a smooth repetitive oscillating pattern), with periods of growth and shrinkage, 


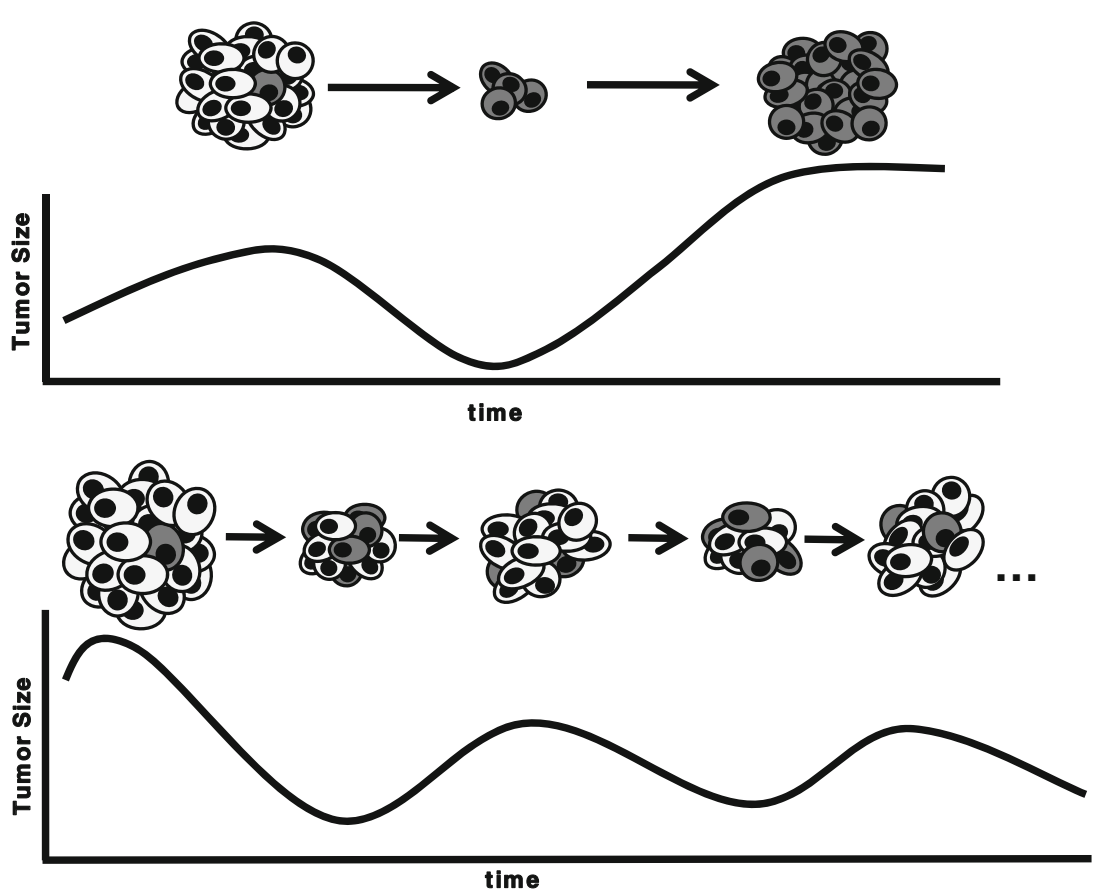

Fig. 2 Top panel. Current chemotherapy regimens initially cause a major reduction of the tumor size, which mostly kills chemo-sensitive cells. This creates a powerful selective pressure that will inevitably benefit clones (shown in dark gray) that have intrinsic resistance to the treatment. Thus, chemo-resistant clones, now unhindered by competition from chemo-sensitive clones, can repopulate the landscape and eventually emigrate to other tissues. Bottom panel. Proposed adaptive cancer therapy, in which the tumor is partially debulked

but not a complete eradication of the tumor mass (Fig. 2, bottom). Interestingly, Gatenby and collaborators show that this approach, at least in a mouse tumor model, provides better survival than the current standard protocols, notably with substantially lower doses of chemo drugs (Gatenby et al. 2009). Thus, although this approach has not been tested clinically, we may be able to harness the inherent competitive nature of cancer cells for patient benefit.

Another approach to cancer treatment that recognizes the adaptive nature of cancer populations is via targeted therapies. Cancer research over the last 50 years has revealed extensive insight into the proteins and enzymes that drive cancer initiation and maintenance, leading to the creation of targeted drugs that inhibit the oncogenic driver (the mutated oncogene that the cancer depends on; Hanahan and Weinberg 2011). Thus, these smart drugs target a small number of proteins in the cell, which contrasts with the scorched-earth approach using DNA-damaging chemotherapies. While the targeted approach has produced some notable successes, such as for chronic myelogenous leukemia, the development of resistance is a substantial problem, particularly for more advanced (and hence complex) cancers (Daley 2003; Bunn 2007). Given that we currently possess a growing list of targeted drugs against specific oncogenes, current approaches would initiate treatment against oncogene A, with a mild chemotherapy regimen but where a portion of the chemo-sensitive population is allowed to survive and oppose the growth of chemo-resistant clones (dark gray cells). Thus, this type of treatment would cause the tumor size to follow a sinusoid growth curve with periods of growth and shrinkage. This approach should control tumor burden but without the goal of complete eradication of the disease

and once resistance and relapse occur due to a mutationactivating oncogene $\mathrm{B}$, the patient would be treated with a drug that would selectively inhibit oncogene B. But if we instead consider evolutionary and ecological principles, and we can recognize that it would be easier to eliminate cancer cells with oncogene $B$ before these cells are selected for and dominate the cancer, we can predict that developing combination therapies up front that target both the driving oncogene and anticipated escape mechanisms will be more effective. But to uncover these escape mechanisms (in essence, before they happen), we have to understand the evolutionary trajectories of cancers under different contexts. If we can anticipate how the cancer in patient 1 with driver oncogenic mutation A will evolve in response to a drug targeting oncogene A, which could be different from the cancer in patient 2 with oncogenic mutation $\mathrm{X}$, then we can better treat each patient. But as we've learned, we must also consider the entire environment for the cancer (the patient), and therapeutic strategies will need to be tailored dependent on the genotype of the patient and many other parameters (age, exposures, etc.).

Finally, using evolutionary principles, can we treat cancer with the goal of avoiding metastatic events? As mentioned before, a tumor is a complex, dynamic system where cells compete for resources, and while increased 
motility should actually be disadvantageous in a homogeneous environment, increased mobility can be advantageous under variably harsh conditions (Chen et al. 2011). Given the dense, cell-compacted nature of tumors, acidic and low oxygen conditions inevitably ensue, which interestingly have been mechanistically shown to promote the evolution of clones which are prone to emigrate from the tumor. In other words, the same environment selects for clones that can depart from the main tumor and establish new colonies in greener pastures. If, for example, we could re-oxygenate tumors and prevent low oxygen conditions, we could theoretically prevent the rise of clones with a more motile phenotype and decrease the possibility of a successful metastatic event. This approach has been tested in animal models (Mazzone et al. 2009), with encouraging results. Thus, understanding cancers from an ecological and evolutionary perspective can lead to innovative approaches to control and target this deadly disease.

\section{Conclusions}

Understanding cancer, both at the tissue and animal levels, demands an evolutionary perspective. The evolution of multicellular animals, particularly those like us with large bodies and long lives, necessitated the acquisition of potent tumorsuppressive mechanisms, which operate at levels of individual cells, tissue organization, and the whole body to limit cancers. Cancer itself follows an evolutionary trajectory, with acquisition of mutations that allow the cancer cells to adapt and succeed, from the initial tiny tumor to a metastatic monster. We have discussed how carcinogenic contexts from chemicals, to radiation, to aging, can promote cancer evolution both by increasing mutation frequency and by promoting selection for adaptive mutations. And like animal populations, cancers respond to insults with selection for the clones that are most fit under that context, such as during chemotherapy. While this view may seem depressing from a therapeutic standpoint, by understanding cancer from an evolutionary and ecological perspective, we should be able to design more rational therapeutic approaches that manipulate cancer's evolutionary trajectories for patient benefit.

Acknowledgments These studies were supported by grants from the National Institutes of Health, the American Institute for Cancer Research, and the Leukemia and Lymphoma Society. We thank Brian Whitney, Carlo Maley, Jennifer Salstrom, and Francesca ÁlvarezCalderón for their critical comments.

\section{References}

Allan JM, Travis LB. Mechanisms of therapy-related carcinogenesis. Nat Rev Cancer. 2005;5(12):943-55.
Bagby GC, Fleischman AG. The stem cell fitness landscape and pathways of molecular leukemogenesis. Front Biosci (Schol Ed). 2011;3:487-500.

Baker NE. Cell competition. Curr Biol. 2011;21(1):R11-5.

Bernards R, Weinberg RA. A progression puzzle. Nat. 2002;418 (6900):823.

Bissell MJ, Hines WC. Why don't we get more cancer? A proposed role of the microenvironment in restraining cancer progression. Nat Med. 2011;17(3):320-9.

Bunn Jr PA. Can acquired resistance to epidermal growth factor receptor tyrosine kinase inhibitors be overcome by different small-molecule tyrosine kinase inhibitors. J Clin Oncol. 2007;25 (18):2504-5.

Cairns J. Mutation selection and the natural history of cancer. Nat. 1975;255(5505):197-200.

Campisi J. Aging, tumor suppression and cancer: high wire-act! Mech Ageing Dev. 2005;126(1):51-8.

Caspari R, Lee SH. Older age becomes common late in human evolution. Proc Natl Acad Sci U S A. 2004;101(30):10895900.

Chan SR, Blackburn EH. Telomeres and telomerase. Philos Trans R Soc Lond B Biol Sci. 2004;359(1441):109-21.

Chen J, Sprouffske K, Huang Q, Maley CC. Solving the puzzle of metastasis: the evolution of cell migration in neoplasms. PLoS One. 2011;6(4):e17933.

Crespi B, Summers K. Evolutionary biology of cancer. Trends Ecol Evol. 2005;20(10):545-52.

Daley GQ. Gleevec resistance: lessons for target-directed drug development. Cell Cycle. 2003;2(3):190-1.

DeGregori J. Evolved tumor suppression: why are we so good at not getting cancer. Cancer Res. 2011;71(11):3739-44.

Dunn GP, Old LJ, Schreiber RD. The immunobiology of cancer immunosurveillance and immunoediting. Immun. 2004;21 (2):137-48.

Farazi PA, DePinho RA. The genetic and environmental basis of hepatocellular carcinoma. Discov Med. 2006;6(35):182-6.

Fleenor CJ, Marusyk A, DeGregori J. Ionizing radiation and hematopoietic malignancies: altering the adaptive landscape. Cell Cycle. 2010;9(15):3005-11.

Frank SA, Nowak MA. Problems of somatic mutation and cancer. Bioessays. 2004;26(3):291-9.

Gatenby RA, Gillies RJ. A microenvironmental model of carcinogenesis. Nat Rev Cancer. 2008;8(1):56-61.

Gatenby RA, Silva AS, Gillies RJ, Frieden BR. Adaptive therapy. Cancer Res. 2009;69(11):4894-903.

Gatenby RA, Gillies RJ, Brown JS. Evolutionary dynamics of cancer prevention. Nat Rev Cancer. 2010;10(8):526-7.

Gorbunova V, Seluanov A. Coevolution of telomerase activity and body mass in mammals: from mice to beavers. Mech Ageing Dev. 2009;130(1-2):3-9.

Gordon MY, Lewis JL, Marley SB. Of mice and men... and elephants. Blood. 2002;100(13):4679-80.

Greaves M. Darwinian medicine: a case for cancer. Nat Rev Cancer 2007;7(3):213-21.

Hanahan D, Weinberg RA. Hallmarks of cancer: the next generation. Cell. 2011;144(5):646-74.

Hecht SS. Cigarette smoking and lung cancer: chemical mechanisms and approaches to prevention. Lancet Oncol. 2002;3(8):461-9.

Hipfner DR, Cohen SM. Connecting proliferation and apoptosis in development and disease. Nat Rev Mol Cell Biol. 2004;5 (10):805-15.

Hoeijmakers JH. DNA damage, aging, and cancer. N Engl J Med. 2009;361(15):1475-85.

Kaplan H, Hill K, Lancaster J, Hurtado AM. A theory of human life history evolution: diet, intelligence, and longevity. Evolut Anthropol. 2000;9(4):156-85. 
Kirkwood TB. Understanding the odd science of aging. Cell. 2005;120(4):437-47.

Liou GY, Storz P. Reactive oxygen species in cancer. Free Radic Res. 2010;44(5):479-96.

Little JB. Radiation carcinogenesis. Carcinogenesis. 2000;21(3):397-404.

Loeb LA, Harris CC. Advances in chemical carcinogenesis: a historical review and prospective. Cancer Res. 2008;68 (17):6863-72.

Lowe SW, Cepero E, Evan G. Intrinsic tumour suppression. Nat. 2004;432(7015):307-15.

Marusyk A, Polyak K. Tumor heterogeneity: causes and consequences. Biochim Biophys Acta. 2010;1805(1):105-17.

Mazzone M, Dettori D, de OR Leite, Loges S, Schmidt T, Jonckx B, et al. Heterozygous deficiency of PHD2 restores tumor oxygenation and inhibits metastasis via endothelial normalization. Cell. 2009;136 (5):839-51.

Medawar P. An unsolved problem of biology. London: H.K.Lewis; 1952.

Merlo LM, Pepper JW, Reid BJ, Maley CC. Cancer as an evolutionary and ecological process. Nat Rev Cancer. 2006;6(12):924-35.

Mortelmans K, Zeiger E. The Ames Salmonella/microsome mutagenicity assay. Mutat Res. 2000;455(1-2):29-60.

Mukherjee S. The emperor of all maladies: a biography of cancer. 1st ed. New York: Scribner; 2010.

Nesse RM. How is Darwinian medicine useful? West J Med. 2001;174 (5):358-60.
Nowell PC. The clonal evolution of tumor cell populations. Sci. 1976;194(4260):23-8.

Pepper JW, Sprouffske K, Maley CC. Animal cell differentiation patterns suppress somatic evolution. PLoS Comput Biol. 2007;3 (12): 250 .

Piazuelo MB, Epplein M, Correa P. Gastric cancer: an infectious disease. Infect Dis Clin North Am. 2010;24(4):853-69.

Raup DM. Biological extinction in earth history. Sci. 1986;231:1528-33.

Reya T, Morrison SJ, Clarke MF, Weissman IL. Stem cells, cancer, and cancer stem cells. Nat. 2001;414(6859):105-11.

Robert J. Comparative study of tumorigenesis and tumor immunity in invertebrates and nonmammalian vertebrates. Dev Comp Immunol. 2010;34(9):915-25.

Sharpless NE, DePinho RA. Telomeres, stem cells, senescence, and cancer. J Clin Invest. 2004;113(2):160-8.

Siegmund KD, Marjoram P, Tavare S, Shibata D. Many colorectal cancers are "flat" clonal expansions. Cell Cycle. 2009;8 (14):2187-93.

Takahashi H, Ogata H, Nishigaki R, Broide DH, Karin M. Tobacco smoke promotes lung tumorigenesis by triggering IKKbeta- and JNK1-dependent inflammation. Cancer Cell. 2010;17(1):89-97.

Weissman IL. Stem cells: units of development, units of regeneration, and units in evolution. Cell. 2000;100(1):157-68.

Williams GC. Pleiotropy, natural selection, and the evolution of senescence. Evol. 1957;11:398-411. 